\title{
Stock Pattern Classification from Charts using Deep Learning Algorithms
}

\author{
Celal Buğra Kaya, Alperen Yılmaz, Gizem Nur Uzun, Zeynep Hilal Kilimci \\ Department of Information Systems Engineering, Kocaeli University, Kocaeli, Türkiye \\ \{celalbugrakaya, alperenylmaz61, gzmnuruzun\}@gmail.com, zeynep.kilimci@kocaeli.edu.tr
}

\begin{abstract}
Pattern classification is related with the automatic finding of regularities in dataset through the utilization of various learning techniques. Thus, the classification of the objects into a set of categories or classes is provided. This study is undertaken to evaluate deep learning methodologies to the classification of stock patterns. In order to classify patterns that are obtained from stock charts, convolutional neural networks (CNNs), recurrent neural networks (RNNs), and long-short term memory networks (LSTMs) are employed. To demonstrate the efficiency of proposed model in categorizing patterns, hand-crafted image dataset is constructed from stock charts in Istanbul Stock Exchange and NASDAQ Stock Exchange. Experimental results show that the usage of convolutional neural networks exhibits superior classification success in recognizing patterns compared to the other deep learning methodologies.
\end{abstract}

Key words: Pattern classification, stock patterns, convolutional neural networks, recurrent neural networks, long short term memory networks.

\section{Introduction}

Classification/categorization is a significant research area due to large amount of images available in diversified types of application fields. Pattern classification is related with the automatic finding of regularities in dataset through the utilization of various learning techniques. Thus, the classification of the objects into a set of categories or classes is provided. Some applications [1-8] of pattern recognition is centered on image classification while patterns are constructed and classified from real data values in some applications [9-12]. In this work, we focus on image-based pattern classification in order to recognize stocks patterns from charts by gathering images of two different patterns. The approach of categorizing images into one of several pre-assigned classes is described as image classification. There are too many application areas such as satellite image classification, medical image categorization, hyperspectral image classification, etc. In image classification field, the explanatory and distinctive sides of attributes evaluated are crucial in order to exhibit good classification success. For this reason, feature extraction techniques are carried out as a separate phase before classifying images when traditional classification models are utilized.

The utilization of deep learning (DL) models has become an attractive in different study fields such as natural language processing, machine translation, image processing, and speech recognition. Deep learning architecture is preferred due to its capability of automatic attribute selection by training more complicated attributes with minimum exterior aid in order to attain significant representation of data through deep neural networks in addition to classification instead of using conventional machine learning algorithms in image classification domain [9-12]. Convolutional neural networks (CNNs), recurrent neural networks (RNNs), long short-term memory networks (LSTMs), deep neural networks (DNN) are well-known deep learning architectures and applied in this work as deep learning algorithms.

In this study, it is purpose to design stock pattern classifier with the help of deep learning

*Corresponding author: Zeynep Hilal Kilimci Address: Department of Information Systems Engineering, Kocaeli University, 41001, Kocaeli, TURKEY. E-mail address: zeynep.kilimci@ kocaeli.edu.tr, Phone: +90 (262) 3032242 
architecture. Thus, extraction of useful information by recognizing patterns from stock charts is provided through deep learning algorithms. For this purpose, convolutional neural networks, recurrent neural networks, and long short-term memory networks are utilized for classifying patterns. Because of the lack of pre-trained dataset for patterns in stock movements, we construct our pre-trained dataset by gathering pattern images from different stock exchange markets. Thus, we construct hand-crafted image dataset from stock charts in Istanbul Stock Exchange and NASDAQ Stock Exchange in order to demonstrate the effectiveness of designed model in classifying patterns. After that we focus on "double-top" and "double-bottom" patterns to extract from stock charts. Then, images are fed into deep learning techniques used in this work in order to classify them. Experiment results present that CNN technique outperforms other with $98.84 \%$ of accuracy.

The rest of paper is established as follows: Section 2 presents a short review of literature works about image and pattern classification tasks. Section 3 introduces deep learning methodologies employed in the experiments. Section 4 gives the details of experiment settings. Section 5 demonstrates the detailed results of experiments. In Section 6, the paper is completed by explaining Conclusion part.

\section{Related Work}

This section gives a summary of mostly deep learning methods such as convolutional neural networks (CNNs), recurrent neural networks (RNN), long-short term memory networks (LSTMs), deep neural networks (DNN) related to pattern classification and image categorization problems. In [11], Li et. al focus on CNN-based medical image classification problem. For this purpose, lung images of patients are evaluated in order to perform classification task. They report that the usage of CNN model for classifying lung disease in images demonstrates remarkable results. In other study [10], authors concentrate on large-scale remote-sensing image classification using convolutional neural networks. In order to show the efficiency of presented model, satellite imagery dataset is constructed with a small amount of correctly labelled data. They conclude that the proposed model present a large amount of context to ensure fine-grained classification maps. In another work [3], convolutional neural networks are employed in order to classify hyperspectral images. In addition to proposed $\mathrm{CNN}$ model, traditional machine learning algorithms and conventional deep learning-based methods are compared in terms of classification performance. Experiment results indicate that the utilization of 5-layered customized CNN technique outperforms others. In study [1], Velay and Daniel evaluate the performance of CNN and LSTM as deep learning algorithms for the recognition of stock historical data. It is aimed to detect a single pattern in the time series and the performance of CNN and LSTM is compared to both the generalization potential of many models and a fixed code algorithm. As a result of study, it is observed that the LSTM model exhibits the best detection rates while 1D and 2D CNN models exhibits the poorest accuracy levels.

In [9], the impact of the recurrent neural networks is investigated on the hyperspectral image classification. For this purpose, Mou et. al develop a new RNN method that can successfully investigates hyperspectral pixels as sequential data and then classify information through network reasoning. Experimental results on three hyperspectral images in the air show that the proposed 
model exhibits competitive performance compared to the others and provides statistically higher accuracy than SVM-RBF and CNN. In addition, they report that the offered network architecture can present a novel perspective for researchers because of the excellent capability of deep recurrent networks for hyperspectral data. In [8], Kamijo and Tanigawa focus on stock price pattern recognition by using recurrent neural networks. It is aimed to appraise the performance of RNN for recognizing stock price-based patterns. An expert called sixteen triangles are extracted from stock price data for all names of companies listed in the first section of the Tokyo Stock Exchange. The model is developed to evaluate the contextual and temporal transition performances and recurrent networks. RNN model successfully performs pattern recognition in fifteen of the sixteen patterns. In [7], Ting et. al concentrate on a model that transforms numerical stock data into sequences. Authors performs sequential and non-sequential association analysis and utilize this analysis in order to classify and predict later price movements. Comprehensive experiments are conducted to evaluate the performance of relational classification techniques and the efficacy of it in-stock mining and inter-stock mining. They conclude the paper that the proposed model is highly effective in forecasting movements of price as well as comprehension the connection between shares from their parent and sub-companies in the same sector.

In another study [2], a novel stock price estimation model based on pattern classification is designed. A probabilistic relaxation-based algorithm is used to categorize probability vectors of patterns concerned to a larger price of share. To confirm the influence of the presented method used in the research, a series of attempts are performed on the real stock price. They inform that the usage of a novel estimation method presents remarkable classification results on pattern recognition. In [6], Lippmann focuses on early pattern classification problems. It is suggested to use artificial neural networks for solving pattern classification problems. It is stated that previous classification models ignore important practical problems and do not easily solve real world problems. As a result of this research, it is emphasized that adaptive non-parametric neural-net classifiers work better on many real-world problems. Moreover, author emphasizes that parametric neural-net classifiers are mostly more successful than traditional Bayesian approaches and provide low error rates. In the study [4], Kiran et. al evaluate five training algorithms of multilayer classifiers in order to recognize patterns. Authors investigate the effect of multilayered perceptrons (MLP) for the recognition of the control graphics pattern (CCP). In other words, the purpose of this study is to appraise the success of training techniques with the most appropriate model for the CCP recognizer. The MLP as a neural network model and a general classifier is employed to categorize seven various types of SPC graphic patterns.

In [12], a model for pattern recognition of control chart in multivariable process is proposed employing artificial neural networks. The proposed model utilizes artificial neural networks (ANNs) to recognize subclasses of patterns of multivariate abnormal, assign responsible variables for the formation of abnormal pattern, and categorize the parameters of abnormal pattern. The success of the model used is appraised utilizing a real case study and implements its tasks for forecast of pattern parameter, favorably. In [5], fingerprint classification is performed by using poincare index control with three main steps. In the first step, data compression is performed according to the direction model and fingerprint images are splitted into $32 \times 32$ sub-regions to attain pattern of direction. In the second step, relaxation softening is implemented with singularity detection and convergence control. In the third step, the individual points determined in the second 
step are re-examined with the poincare index and classified according to this index. The study is concluded that the proposed approach is able to classify fingerprint patterns into more than ten classes.

Unlike aforementioned studies, this study is undertaken to evaluate deep learning methodologies to the classification of stock patterns from stock charts. Moreover, the lack of pre-trained data in the literature, we construct the dataset that is composed of "double-top" and "double-bottom" patterns by gathering stock images from Istanbul Stock Exchange and NASDAQ Stock Exchange.

\section{Deep Learning Techniques}

In this work, we use three different deep learning techniques for pattern classification task. These are convolutional neural networks (CNNs), Recurrent Neural Networks (RNNs), and Long ShortTerm Memory Networks (LSTMs).

\subsection{Convolutional Neural Networks (CNNs)}

CNNs as one of class of deep learning methodologies have represented remarkable outcomes in image recognition and classification [13-16]. CNN is a version of feed-forward neural network with different layers such as input-output layers, convolution, and pooling layers. CNN method covers a set of convolutional layers associated with pooling layers, and dense layers. The convolution layer is the brain of $\mathrm{CNN}$ architecture. Feature map is acquired among too many features by performing convolution filter. Through the series of filters, the meaningful representation of the features is obtained for any domain. Thus, the demonstration of dataset with the operation of convolution presents dependencies, local information, or semantics hidden among features in the dataset. After that, the most meaningful information is attained through a pooling layer by decreasing the number of instances. For this purpose, down-sampling approach is carried out to reduce the training time, dimension of data, and over-fitting. In Figure 1, the architecture used in this study is demonstrated.

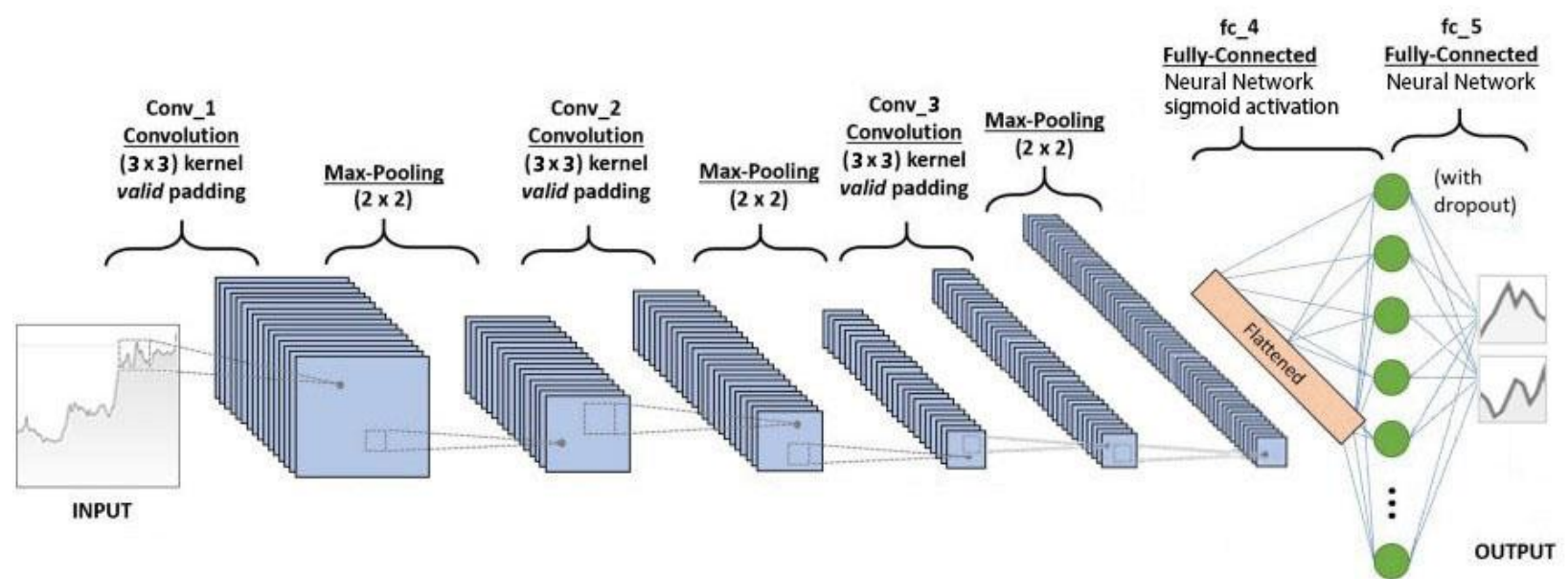

Figure 1. Flowchart of the proposed system 
Max pooling, average pooling, global max pooling, global average pooling are the approaches of pooling layer. In this way, feature extraction step is performed through this characteristic of CNN until here. After, output is firstly converted to the expected input and fed into the fully connected layers in order to perform classification task. To avoid over-fitting, regularization methods can be applied. In Figure

\subsection{Recurrent Neural Networks (RNNs)}

RNNs are preferred in order to shape sequential data. RNNs are defined as a state of hidden functions which are fed from prior hidden states of recurrent nets [17-18]. The output of RNN is based on both current input of the system and former information that is hold in hidden states through gates. There is a difference between RNN, and feed-forward neural network based neural network models in that preceding information is hold in the network. However, there are gradient vanishing or exploding problems in term of finding long-term dependencies in the sequential data. To cope with vanishing or exploding issues, different techniques are employed such as clipping of gradient or appropriate activation functions is utilized.

\subsection{Long Short-Term Memory Networks (LSTMs)}

LSTMs are put forward as a solution of vanishing or exploding issues of RNNs. Besides, LSTMs are also known as customized version of RNNs [19-21]. Essentially, LSTMs are improved in order to attain long-term dependencies that is the missing side of RNNs. Long short-term memory networks propose to hold the long dependencies among data and keep the contextual semantics of information. To hold the dependencies among information in a long range, specialized memory cells are employed. Each unit of LSTM includes gates with different purposes. Input, output and forget gates facilitate remembering, forgetting, or passing information. Thus, LSTM units are capable keep, read, write, and remove information through the usage of gates.

\section{Experiment Setup}

Dataset employed in the experiments are picked up through stock certificate graphs of companies yearling on the website of TradingView [22]. On the hand-building collected graphs, datasets are constituted by searching for "double-bottom" and "double-up" patterns. Thus, categories are determined as "double-bottom" and "double-top", separately. Before the data sets are fed into the deep learning models, tagging process is implemented. As a result, 3,242 instances are generated totally as 1,542 of them are "double-bottoms" patterns and 1,697 of them are "double-top" patterns. After that, all stock patterns are given as input into the deep learning algorithms in order to observe classification performances of each deep learning model. Dimensions of inputs are arranged as 100 height, 30 width gray cmap. Dataset is converted into vector by utilizing of cv2 library.

Comprehensive experiments are carried on the Jupyter notebook using Python language with the latest version. Deep learning models in this study are trained on Google Colab [23] platform using CPU and GPU hardware. The number of neurons of 3-layer model structures are set to be 16-3264 and the batch length, which is one of the training parameters, is fixed to 64. In addition, a number of procedures are performed to ensure that training models give better accuracy and prevent overfitting. In order to avoid over-fitting issue which consists during the model training, dataset is scaled interval between 0 and 1 by providing normalization. Therewithal, dropouts are added to 
the layers and some of the neurons are ignored during training to decrease overfitting. CNN performs faster training time than both RNN and LSTM. The reason why RNN and LSTM are close to each other in terms of training time because LSTM is actually another revised version of RNN. As a result of the experiments, it is observed that as the number of epoch increases, the loss value decreases and the accuracy value increases. As another observation, in the 15 th epoch for the CNN model, training accuracy reaches the maximum level and loss value reaches minimum level. For this reason, continuing training after the 15th epoch does not make sense in terms of accuracy rate and loss value.

The most important problem is longer training time during the training procedure because experiments are carried out on CPU, initially. Then, GPU is employed instead of CPU to reduce training time duration. Moreover, 4-dimensional input structures are transformed into the 3dimensional by using reshape function for differences between input dimensions of LSTM ,RNN and dimension of CNN. The other way for getting the most efficient classification performance is diversification of layers and activation functions. While the best accuracy is provided with rectified linear unit ( $\mathrm{ReLu}$ ) activation function for $\mathrm{CNN}$, Tanh activation function gives the best accuracy performance for LSTM and RNN.

\section{Experiment Results}

The objective of this work is to categorize stock patterns from charts using deep learning methodologies. Convolutional neural networks (CNNs), recurrent neural networks (RNNs), longshort term memory networks (LSTMs) are used in the experiments. The experiment results are indicated in Table 1 and Table 2, respectively. Training set percentage and epoch size are abbreviated as "ts" and "es", respectively. CNN, RNN, and LSTM are the abbreviation of deep learning algorithms located in the tables. The best accuracy value is indicated as bold letters in tables.

Table 1. Classification accuracies of deep learning algorithms at all training set sizes.

\begin{tabular}{cccc}
\hline $\begin{array}{c}\text { Training Set } \\
\text { Size }\end{array}$ & CNN & RNN & LSTM \\
\hline 80 & 95.99 & 83.07 & 87.40 \\
70 & 95.79 & 82.65 & 82.12 \\
50 & 93.40 & 79.33 & 74.70 \\
30 & 90.13 & 55.00 & 51.70 \\
10 & 86.20 & 48.47 & 51.74 \\
\hline avg & $\mathbf{9 2 . 0 3}$ & 69.70 & 69.53 \\
\hline
\end{tabular}

In Table 1, classification performances of deep learning algorithms are presented in terms of classification accuracies by varying training set percentages. When deep learning algorithms are considered, $\mathrm{CNN}$ exhibits superior classification performance compared to the others at all training set percentages. Except training set size 10, CNN technique performs approximately $90 \%$ of accuracy value. The best accuracy value is observed as $95.99 \%$ at ts 80 . It is followed by $95.79 \%$, $93.40 \%, 90.13 \%, 86.20 \%$ accuracy results at ts 70 , ts 50 , ts 30 , ts 10 , respectively. To put it another way, if training set size increases, the accuracy value generally increases and vice versa. In addition, mean accuracy value of $\mathrm{CNN}$ is observed as $92.03 \%$ of accuracy. Considering the experiment results for RNN, the best accuracy value is ensured as $83.07 \%$ of accuracy at ts80 while 
the poorest accuracy result which is $48.47 \%$ of accuracy is observed where training set percentage is 10. When mean accuracy results are considered, it is clearly observed that RNN exhibits approximately $23 \%$ decrease in classification success compared to CNN. At ts80 and ts70, CNN outperforms RNN by providing nearly $12 \%$ improvement. At lower training set percentages such as ts 30 and ts 10 , remarkable decrease in classification accuracies is observed from $79.33 \%$ of accuracy at ts 50 to $55.00 \%$ at ts 30 and $48.47 \%$ at ts 10 .

The best accuracy value of LSTM is monitored as $87.40 \%$ of accuracy at ts 80 . It is ordered by from the best to the worst accuracy results as $82.12 \%, 74.70 \%, 51.74 \%, 51.70 \%$ of accuracies at in order of ts70, ts50, ts10, ts 30 . When the average accuracy result is taken into account, the worst classification success is attained with $69.53 \%$ of accuracy compared to CNN and RNN. It is clearly seen at all training set size that classification results of RNN and LSTM are competitive and very close to each other. Therefore, the difference in classification success between RNN and LSTM can be negligible. The proximity in classification accuracies between RNN and LSTM is expected because LSTM is the specialized version of LSTM. Furthermore, classification accuracies od CNN exhibits superior performance at all training set sizes compared to LSTM. The mean classification accuracies of the deep learning algorithms are obtained $92.03 \%$ for CNN, $69.70 \%$ for RNN, and $69.53 \%$ for LSTM. Thus, the order success of deep learning algorithms can be summarized as CNN> RNN> LSTM when average accuracy values are considered. Classification accuracies of each deep learning techniques boosts as training set sizes increase except LSTM at ts10. Because the classification success of LSTM demonstrates almost similar performance passing from ts 10 to ts 30 . As a conclusion of Table 1, the best classification results are attained at ts 80 while the poorest accuracy values are generally obtained at ts10 as expected. Moreover, these results are achieved in the experiments when epoch size is adjusted to 50 .

Table 2. Classification accuracies of deep learning algorithms at all training set sizes.

\begin{tabular}{cccc}
\hline Epoch Size & CNN & RNN & LSTM \\
\hline 25 & 95.99 & 87.91 & 51.39 \\
50 & 99.23 & 80.00 & 74.70 \\
75 & 99.54 & 83.09 & 85.06 \\
100 & 99.69 & 88.22 & 84.14 \\
150 & 99.98 & 90.68 & 88.39 \\
200 & 99.23 & 88.86 & 84.65 \\
\hline avg & $\mathbf{9 8 . 8 4}$ & 86.46 & 78.05 \\
\hline
\end{tabular}

In Table 2, classification performances of deep learning algorithms are presented by diversifying epoch sizes. Experiment results located in Table 2 is prepared thereby fixing training set size to 80 because of its superior performance compared to the other training set percentages. The poorest classification accuracy is seen for CNN as $95.99 \%$ of accuracy and for LSTM as $51.39 \%$ of accuracy at es 25 while the worst classification performance is observed for RNN as $80.00 \%$ of accuracy at es50. When average accuracy values are considered, CNN remarkably outperforms other deep learning techniques by acquiring almost $12 \%$ and nearly $20 \%$ enhancements compared to RNN and LSTM, respectively. As a result of Table 2, CNN displays outstanding classification performance in terms of accuracy values at all epoch sizes. While the highest accuracy value of 
RNN is appointed as $90.68 \%$ at es 150 , the lowest accuracy value is attained as $80.00 \%$ percent at es50.

As a different from CNN, while RNN passes through from es 25 to es50, accuracy value decreases as nearly $7 \%$. Therefore, the difference in classification accuracies between CNN and RNN is rather different at es50. Because CNN exhibits approximately 4\% improvement while RNN shows almost $7 \%$ decrement in classification success when passing through es50 from es 25 . Consequently, RNN presents $86.46 \%$ of accuracy as a mean classification accuracy which is nearly $12 \%$ lower than the average accuracy value of CNN. The classification success of RNN can be preferable compared to LSTM model because of $8 \%$ improvement of RNN in classification success of the system. On the other hand, the poorest accuracy value is $51.39 \%$ at es 25 for LSTM technique similar to CNN. The best performance for LSTM algorithm is presented as $88.39 \%$ at es150 and $88.86 \%$ of accuracy at es 200 . This means that LSTM model reaches the maximum accuracy values when epoch size is set to 200 . Then, training procedure can be stopped for the latter epoch sizes considering validation accuracies in order to avoid over-fitting. As a result of Table 2, it is clearly seen that the impact of epoch sizes in average classification accuracies can be summarized as: CNN $>$ RNN $>$ LSTM. When the impact of training set sizes and epoch sizes are evaluated, the best classification results are attained with 150 epochs and ts80 for all deep learning algorithms.

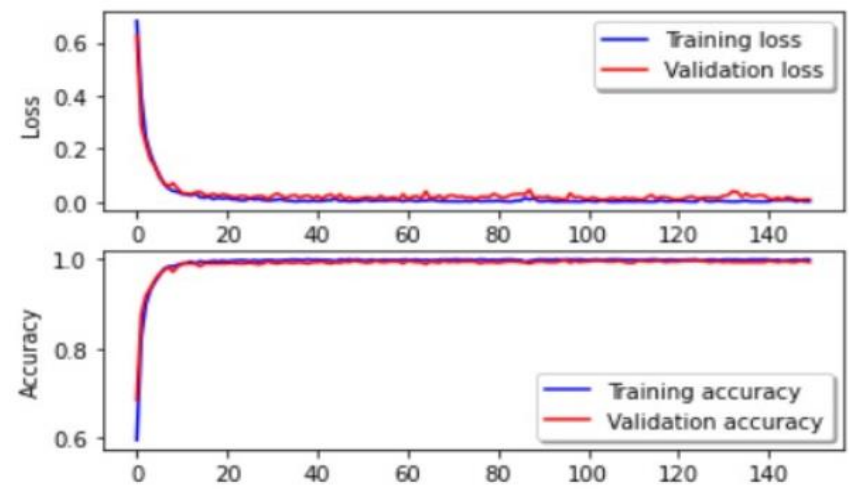

Figure 1. Classification Accuracy And Loss Results Of CNN At 150 Epochs And Ts80.

In Figure 1, accuracy and loss values are given for CNN whose classification success is the best in this work. It is clearly observed that accuracy values increase when epoch size is also increased. However, the classification performance is stable after epoch size 15 which means that training procedure can be stopped at es 15 as a stop criterion.

\section{Discussion and Conclusion}

In this work, stock pattern classifier is designated with the help of deep learning architecture. Thus, recognizing of beneficial information by extracting patterns from stock charts is ensured through deep learning techniques. For this objective, convolutional neural networks, recurrent neural networks, and long short-term memory networks are appraised for categorizing patterns. Because of the lack of pre-trained dataset for patterns in stock movements, we construct our pre-trained dataset by collecting images of patterns from different stock exchange markets. Thus, we construct hand-crafted image dataset from stock charts in Istanbul Stock Exchange and NASDAQ Stock 
Exchange in order to show the efficacy of designed model in categorizing patterns. Then, we concentrate on "double-top" and "double-bottom" patterns to extract from images of stock charts. After that, images are fed into deep learning techniques employed in this study to categorize them. Experiment results present that CNN technique outperforms the other models with $98.84 \%$ of accuracy.

\section{References}

[1] M. Velay and F. Daniel "Stock chart pattern recognition with deep learning." arXiv preprint arXiv:1808.00418, 2018.

[2] Z. Zhanggui, H. Yau and A. M. N. Fu, "A new stock price prediction method based on pattern classification," IJCNN'99. International Joint Conference on Neural Networks. Proceedings (Cat. No.99CH36339), pp. 3866-3870, vol.6, 1999.

[3] T.T. El-Midany, M. A. El-Baz, and M. S. Abd-Elwahed. "A proposed framework for control chart pattern recognition in multivariate process using artificial neural networks." Expert Systems with Applications 37.2 2010, pp. 1035-1042.

[4] I. Kiran, M.P. Devi, and G.V. Lakshmi. "Training Multilayered Perceptrons for Pattern Recognition: A Comparative Study of Five Training Algorithms." World Congress on Engineering 2012. July 4-6, 2012. London, UK.. Vol. 2188. International Association of Engineers, 2010.

[5] M. Kawagoe and A. Tojo. "Fingerprint pattern classification." Pattern recognition 17.3 ,1984, pp. 295-303.

[6] R. P. Lippmann, "Pattern classification using neural networks," in IEEE Communications Magazine, 27.11, pp. 47-50, 1989.

[7] J. Ting, T. Fu, and F. Chung. "Mining of stock data: intra-and inter-stock pattern associative classification." Threshold 5.100, 2006, pp. 5-99.

[8] K. Kamijo and T. Tanigawa, Tetsuji. "Stock price pattern recognition-a recurrent neural network approach." 1990 IJCNN International Joint Conference on Neural Networks. IEEE, 1990.

[9] L. Mou, P. Ghamisi and X. X. Zhu. "Deep recurrent neural networks for hyperspectral image classification." IEEE Transactions on Geoscience and Remote Sensing 55(7), 2017, pp.3639-3655.

[10] E. Maggiori, Y. Tarabalka, G. Charpiat and P. Alliez. "Convolutional neural networks for large-scale remote-sensing image classification." IEEE Transactions on Geoscience and Remote Sensing 55 (2), 2016, pp. 645-657. 
[11] Q. Li, W. Cai, X. Wang, Y. Zhou, D.D. Feng, \& M. Chen,. "Medical image classification with convolutional neural network." 2014 13th International Conference on Control Automation Robotics \& Vision (ICARCV). IEEE, 2014.

[12] W. Hu, et al. "Deep convolutional neural networks for hyperspectral image classification." Journal of Sensors 2015, 2015.

[13] J. Schmidhuber, "Deep Learning in neural networks: An overview," Neural Networks, vol. 61, pp. 85-117, 2015.

[14] G. H. Yann LeCun, Yoshua Bengio, "Deep learning (2015), Y. LeCun, Y. Bengio and G. Hinton," Nature, 2015.

[15] A. Voulodimos, N. Doulamis, A. Doulamis, and E. Protopapadakis, "Deep Learning for Computer Vision: A Brief Review," Comput. Intell. Neurosci., 2018.

[16] A. Krizhevsky, I. Sutskever, and G. E. Hinton, "ImageNet Classification with Deep Convolutional Neural Networks," in ImageNet Classification with Deep Convolutional Neural Networks, 2012.

[17] Z. C. Lipton, J. Berkowitz, and C. Elkan, "A Critical Review of Recurrent Neural Networks for Sequence Learning," May 2015.

[18] J. L. Elman, "Finding structure in time," Cogn. Sci., 1990.

[19] K. Greff, R. K. Srivastava, J. Koutnik, B. R. Steunebrink, and J. Schmidhuber, "LSTM: A Search Space Odyssey,” IEEE Trans. Neural Networks Learn. Syst., 2017.

[20] D. Kent and F. M. Salem, "Performance of Three Slim Variants of The Long Short-Term Memory \{(LSTM)\} Layer," CoRR, vol. abs/1901.00525, 2019.

[21] C. Olah, "Understanding LSTM Networks," colah.github.io, 2015

[22] https://www.tradingview.com/

[23] colab.research.google.com 